International Journal of Wireless \& Mobile Networks (IJWMN) Vol. 6, No. 4, August 2014

\title{
Highly Reliable Multi-Service Provisioning Using SeQuential PREdiction OF ZONE AND PL\&T OF NODES IN MOBILE NETWORKS
}

\author{
Sharmistha Khan ${ }^{1}$, Dr. Dhadesugoor R. Vaman ${ }^{2}$, Siew T. Koay ${ }^{3}$ \\ ${ }^{1}$ Doctoral Student, Electrical and Computer Engineering Department and ARO CeBCom, \\ Prairie View A\&M University, Prairie View, TX, USA \\ ${ }^{2}$ Texas A\&M University System Regents Professor and Director of ARO CeBCom, \\ Electrical and Computer Engineering Department, Prairie View A\&M \\ University, Prairie View, TX, USA \\ ${ }^{3}$ Professor, Electrical and Computer Engineering Department, Prairie View A\&M \\ University, Prairie View, TX, USA
}

\begin{abstract}
In this paper, a dynamic Position Location and Tracking (PL\&T) method is proposed which uses an integrated "zone finding by predictive algorithm" and "Triangulation with dynamic reference positioning" for target nodes. The nodes can be used as references or targets at different instances of time to maintain accurate PL\&T of each node in a wireless mobile network. Each node is equipped with an omnidirectional antenna. It is shown that while "zone prediction" is fairly accurate for finding the PL\&T of a target node based on previous good location data when the target is moving linearly in one direction, it is not adequate when the nodes change directions. However, once the zone is predicted, the triangulation will provide accurate position of the target node by placing the reference nodes to form a reasonably perfect geometry. The results show that triangulation alone does not provide accurate prediction of the target location without finding the zone of the target and zone finding alone does not support accuracy of PL\&T when the target node is changing directions. Another aspect that should be of concern is the repeated PL\&T operation on the same target node at different instance of time becomes erroneous and therefore, they need to be reinitialized to get accurate locations with known wait points. Also, the references need to be reinitialized to maintain the accuracy. This requires the nodes to be dynamically used as target nodes and with good PL\&T, they are used as references at different time instances. There is an upper layer dynamic reference management is required for this technology. The proposed integrated predictive zone finding and triangulation is particularly suitable for clustered Mobile Ad hoc Networks (MANET). Finally, this paper shows the performance analysis of this system for a reasonable number of nodes within the cluster that demonstrates high accuracy of PL\&T location data of the nodes. Of course the overhead in managing the dynamic references is increased. However, the frequency of changing the references is minimized in each cluster as it contains few nodes (40 or less).
\end{abstract}

\section{KEYWORDS}

Position, Localization, Tracking, Zone Prediction, Triangulation

\section{INTRODUCTION}

Currently, a variety of wireless technologies are rapidly emerging for supporting multi-service provisioning. These technologies include: Wireless Metropolitan Area Networks (WMAN), Wireless Local Area Networks (WLAN), Wireless Wide Area Networks (WWAN), the Worldwide Interoperability for Microwave Access (WiMAX), General Packet Radio Service 
International Journal of Wireless \& Mobile Networks (IJWMN) Vol. 6, No. 4, August 2014

(GPRS), Universal Mobile Telecommunications System (UMTS), and Wireless Fidelity (WiFi). They are being deployed as part of the overall Internet Protocol (IP) based infrastructure and are standardized to provide high speed data rate, high-quality, and high capacity multimedia services in different geographic areas. They also support mobility whereby users can access the network centric applications wherever they have access to these networks and not limited to their home base. In addition, the deployment of backhaul wireless networks is facilitating the users to have greater access broadband multi-services applications. However, in mobile communication uninterrupted network connectivity is still challenging. It is really essential to provide continuous network connectivity to satisfy high levels of mobile service quality and maintain the prescribed levels of security and privacy. The most challenge today in wireless network research is to support end-to-end quality of service to a wide variety of multi-services that includes both real time and non-real time applications. To provide continuous network connectivity, it is very essential to get information about the required transmission power for maintaining the connectivity between a source and a destination and their location identification to support better management for connectivity. The Position, Location and Tracking (PL\&T) of a node needs to be accurately maintained at all time. Target or object (or node) PL\&T has become critical in many applications such as navigation, tracking, emergency service, location based services (LBS) and security. Several algorithms have been already built for tracking the target node accurately. Most of them have used the received signal power methodology. However it is really difficult to find out the location of a target accurately as the received signal tends to be distorted due to jitter, channel noise, and interference. Therefore, still some improvement and innovation is required to achieve the PL\&T accuracy.

In this paper, we propose a PL\&T algorithm based on a zone prediction and triangulation that allows the nodes to use omnidirectional antennas. Tracking accuracy needs to be maintained to ensure power efficiency and connectivity at all times. Currently most of the commercial systems use Global Positioning System (GPS) for node PL\&T while it is moving. However, GPS algorithms tend to be inaccurate near buildings and when the nodes are not visible due to weather or blocked areas. GPS also cannot be used indoors to predict PL\&T as it does not distinguish different floor [1]. In this paper, we propose to use nodes which have accurate PL\&T at an instance of time as references for triangulation which is applied after we predict the zone of the target node. The zone prediction prior to triangulation allows the reference nodes to be places to form a good geometry for triangulation to improve the PL\&T accuracy. Our proposed algorithm will able to track any moving objects as well as stationary objects. Our algorithm does not restrict the directional movement of the target nodes. The performance of the combined zone prediction and triangulation will be demonstrated for its accuracy.

In zone prediction algorithm, the target location at an instance of time is predicted based on previous good locations using an iterative process. Once the zone of the target is predicted with respect a relative origin, the nodes that have accurate PL\&T will be used as reference nodes to form a geometry and triangulation will be performed to accurately predict the PL\&T of the target node at that instance of time. As the target node keeps moving (in any direction), the reference nodes will be dynamically changed to maintain proper geometry for computation of PL\&T of the target node at each instance of time after the zone is predicted.

\section{BACKGROUND}

The demand of uninterrupted services is rapidly increasing with the current growth of smart mobile devices, multimedia services such as audio- video streaming, and different location based services .To provide the uninterrupted network services, Position, Location and Tracking (PL\&T) of mobile nodes in a network has become a very important methodology as an alternate method of using power measurements. Average power measurements are impacted by the interference such 
as multi-fading, whereas PL\&T of nodes with good measurements is more reasonable in assessing the location of a node with respect to a sending node in order to maintain power efficiency and also connectivity between nodes. The use of PL\&T technique has become very useful in both homeland and battlefield theaters to avoid friendly casualties. As long as the accuracy of PL\&T of nodes is maintained within reasonable error, this technique is also very useful in applications where fire hazards may occur and saving life within a constrained time is very critical. Finally, it is possible to see that PL\&T technique has been used in numerous applications today including WSN, navigation, tracking, indoor positioning, emergency 911 in USA, and emergency 112 in Europe.

Researchers have developed various PL\&T algorithms as can be seen in published literature. However, accuracy of these algorithms in tracking moving nodes is still a challenge in many applications. Until, one can develop very accurate predictions using PL\&T, their applications for mission critical areas will be difficult.

To identify the PL\&T of a target, different PL\&T techniques have been developed using friendly reference nodes: single reference node based PL\&T [2], two reference nodes based PL\&T [3], three reference nodes based Triangulation [4], and four reference nodes based multilateration methods and multiple targets' PL\&T [4] where each of above methods is analysed by developing theoretical models for PL\&T precision. Some of the important PL\&T methods are reviewed through literature are described below.

Forward Movement based Prediction: We have found that in this method, only zone prediction method is focused within a constrained forward movement of a target node. It is based on single reference node that considers limited random movement and does not consider sharp turns or obstacles [5].

Cooperative Indoor Position Location technique: This is based on Parallel Projection Method and designed for collaborative position location to effectively handle Non Line Of Sight (NLOS) propagation based on set theory (specifically on the parallel projection method). This method is not applicable for mobile node as well as for MIMO technology. More time consuming as it is based on set theory; it will take additional time to achieve satisfactory level of accuracy [6].

Received Signal Strength based Prediction: This method provides better tracking accuracy over the triangulation method as long as multi-path fading is small and longer averaging availability. However, without zone finding, the signal is spread and errors are accumulated in the computation of both the distance and the angle for limited non-random trajectory [7].

Multi hop based Prediction: All the position locations of nodes are estimated using multiple levels of reference nodes which Increases cumulative errors in multi hop measurements and has no beam adaptation used. Thus, significantly reduces accuracy of tracking [8].

Directional Lines Intersection based Prediction: Localization of nodes using point of intersection of highly directional beams has better accuracy for low speed mobile anchor nodes. In this method, higher overhead in scanning and does not address random trajectory [9].

Distributed Position Localization and Tracking (DPLT) Method: It is a robust, reliable, and low complexity method of Distributed PL\&T detection of malicious nodes in a Cluster based MANET is proposed [4]. However, only forward movement is considered for determining the adaptive beam formation.

By considering the major limitations of all the above methods, we have proposed this novel PL\&T method that uses IP based triangulation based on zone prediction method where accuracies 
International Journal of Wireless \& Mobile Networks (IJWMN) Vol. 6, No. 4, August 2014

of less than a meter are required. However, multi-path fading and noise handling was a serious issue. In this method, nodes require re-initializing after repeated tracking at different instances of time to maintain the tracking error within the specified target. The reference nodes also are changed dynamically as the target nodes move. Therefore, inter-changing the reference nodes and target nodes was dynamically accomplished to maintain accuracy. Thus, this method may increase the overall complexity. On the other hand, multi-path fading and Doppler effects play a significant role in reducing the accuracies of the PL\&T. Many researchers are addressing these issues through equalization, estimation and coding techniques [10-11]. Some researchers use directional antenna which basically allows concentration of beam power in one direction in order to increase the signal strength and increase the probability of handling interferences and improve the PL\&T accuracy [12]. Researchers have also used Directional Antennas in interacting of steered or switched antenna systems in an ad hoc network [13]. However, the main limitation on using the directional antenna is the incapability on using for Multiple Input Multiple Output (MIMO) technology. Again, it is very difficult to use directional antenna in the end user's device. In recent years, in wireless communication field, for getting satisfactory performances, significant progress has been made in developing the overall systems. One of the most beneficial is the use of multiple antennas at the transmitter and at the receiver [14] which known as multiple input multiple output (MIMO) systems. Over using the single input single output (SISO) system, there are mainly two types of benefits on using MIMO system as well as on using multiple antesnnas: spatial diversity improvement and throughput [15]. Therefore, omnidirectional antenna is really beneficial for mobile and wireless devices those have MIMO technology as it can provide a 360 degree horizontal radiation pattern [16]. Considering this issue, we have used omnidirectional antenna in this research.

\section{SYSTEM ARCHITECTURE}

The proposed design of PL\&T system uses a sequential process of "zone finding" followed by placing references to locate the node with a reasonable geometry that minimizes the error in triangulation with minimal outliers. In this design, we quantize a time such that the computational time for integrated zone finding and triangulation of a specific location is significantly smaller and allows the node to be virtually stationary for computation. The next location will be identified at a periodic quantized time. This process does not take into account the location in between two quantized times. It is anticipated that the importance of quantizing the time will allow computational accuracy and at the same time the transient period is not critical for the overall tracking of the mobile node. PL\&T is a continuous operation of finding the location of the node with successive zone finding, placing the references for proper triangulation geometry and performing the triangulation in an atomic operation. Our proposed algorithm will able to find out the location of a target for any direction continuously since it can track the location based on both zone prediction and triangulation method. If a target node moves linearly, through zone prediction method we can predict the location accurately. However, on the other side, when a target does not move linearly means changes it's direction such as though spiral way, trapezoidal way, it required triangulation method with zone prediction to track the location accurately. The proposed algorithm that involves several steps with design architecture is described through Figure 1. Figure 1 shows the basic design architecture of our PL\&T system for a particular scenario where we consider target node moves linearly. 


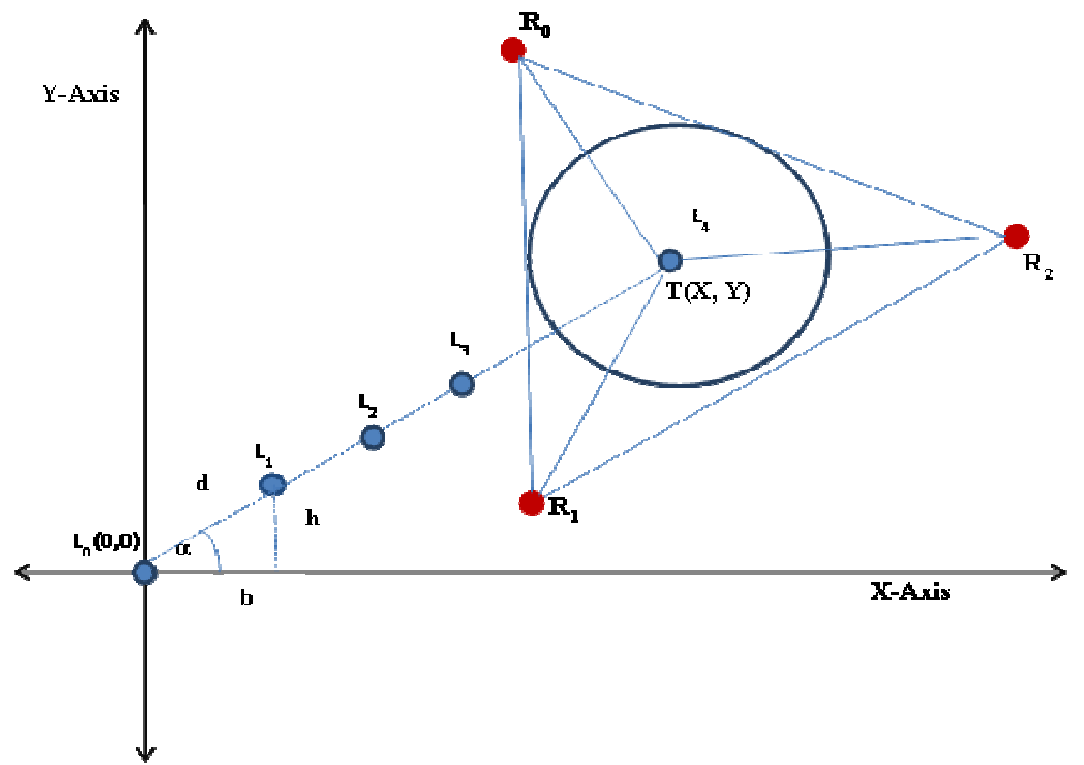

Figure 1. System Architecture of proposed PL\&T

The scenario consist of four mobile nodes where $R_{0}, R_{1}, R_{2}$ are the three reference node and $T(X$, $\mathrm{Y}$ ) is the target node. $\mathrm{L}_{0}, \mathrm{~L}_{1}, \mathrm{~L}_{2}, \mathrm{~L}_{3}$ shows the first four location point of the target node and the circle around the target node whose radius is the half distance between third and fourth point shows the predicted zone of the target node's location point . Based on previous four locations point, we have to find out the current location of a target continuously. Each time we have to find out the predicted zone of the target node's location and after finding the zone, we have to place three reference nodes $\left(R_{0}, R_{1}, R_{2}\right)$ that form three triangles $\Delta R_{0} T R_{2,} \Delta R_{0} T R_{1,}, \Delta R_{1} T R_{2}$, with the target node for performing triangulation process.

\subsection{Zone Finding}

In this research, Zone finding is one of the important processes that basically predict the zone of a target's location of a particular instance of time. To implement this process we need to do the following steps that are described below in details.

\subsubsection{Develop Zone Finder (ZF) algorithm}

Basically, ZF algorithm is an iterative process that helps to find out some previous good location of a target node with respect of an origin. Basic system configuration of $\mathrm{ZF}$ algorithm is presented by Figure 2 which mainly shows the relationship between different elements of this system and their connectivity with each other and consists of a recursive polynomial executor process, mapping and correction algorithm, transport function and database for reporting data. For identifying the next location based on previous four locations, a recursive polynomial executor is used that is shown in Figure 2. IP time tag approach is used by geometric pairing to measures the distances and corrects to match the object location to a common vertex in two or more triangles. The number of triangles those are used in this algorithm is directly proportional to the number of reference points used. The minimum requirement of reference points is two for making a geometric pairing triangulation. For improving the accuracy of tracking objects, the first level of adjustment is provided by this geometric pairing triangulation. For getting high accuracy $(>>1$ meter) in object tracking, an independent second level verification is provided by the $\mathrm{ZF}$ polynomial. 


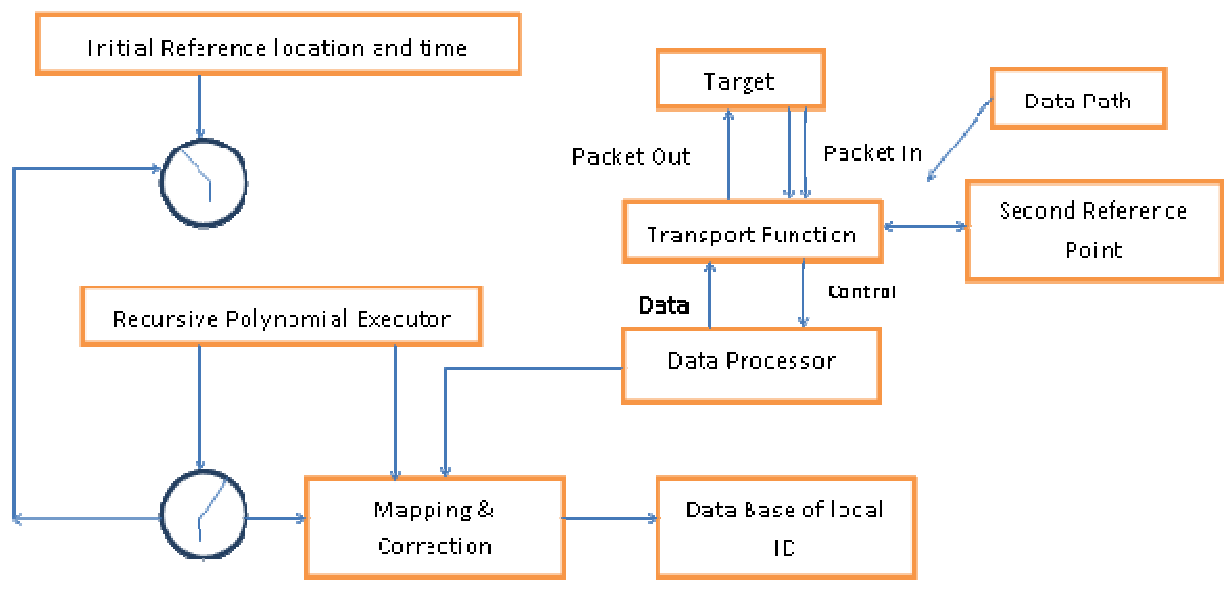

Figure 1. ZF Reference System Configuration

Furthermore, the distances and maps are used to coordinates that are consistent with the GPS coordinates by the reference system configuration. By using a basic IP transport system, within any network centric environment this data can be used for transporting purposes. IP transport is the basic transport method for exchanging data. An IP address is uses as the ID of the object and that can be changed to any customer combat ID.

\subsubsection{Draw the Circle}

By implementing the ZF algorithm, we can get the distance between third and fourth location point. We have considered the fourth location point as the target location. To find out the coordinate of the fourth location point we have to assume some measurement that is shown in Figure 1.

We can assume,

- The co-ordinate of first location point is $\mathrm{L}_{0}(0,0)$.

- Let, the first location point makes angel $\theta$ with the X Axis through its true path.

- From ZF algorithm (We have discussed above), we have the trajectory locations (the distance between one point to another point) of a node for an instance of time. As we have the distances between two consecutive location points $\left(1^{\text {st }}\right.$ location point to $2^{\text {nd }}$ location point and $2^{\text {nd }}$ to $3^{\text {rd }}$ location point), by applying the trigonometric formula, we can get the co-ordinate of the $2^{\text {nd }}$ point, $3^{\text {rd }}$ point, and $4^{\text {th }}$ point accordingly.

We can find out the value of $\mathrm{X}$-axis and $\mathrm{Y}$-axis by the following equations where $\mathrm{b}$ is the coordinate value of $\mathrm{X}$-axis, $\mathrm{h}$ is the coordinate value of $\mathrm{Y}$-axis and $\mathrm{d}$ is the distance between two consecutive location points.

$$
\begin{aligned}
& \cos \theta=\frac{h}{d} \ldots \ldots(3.1) \\
& b=d \cos \theta \ldots \ldots(3.2) \\
& \sin \theta=\frac{h}{d} \ldots \ldots(3.3) \\
& h=d \sin \theta \ldots \ldots(3.4)
\end{aligned}
$$


After getting the co-ordinate of the $4^{\text {th }}$ location point, we have to draw a circle around the $4^{\text {th }}$ point with a particular radius where the value of the radius will be the half distance between $3^{\text {rd }}$ and $4^{\text {th }}$ point. Basically, we will consider the perimeter of this circle as a zone for the target.

\subsubsection{Place Reference Point}

We have to place three reference points those have the accurate PL\&T such as $\mathrm{R}_{0}\left(\mathrm{X}_{0}, \mathrm{Y}_{0}\right)$, $\mathrm{R}_{1}\left(\mathrm{X}_{1}, \mathrm{Y}_{1}\right), \mathrm{R}_{2}\left(\mathrm{X}_{2}, \mathrm{Y}_{2}\right)$ around the target node such a way that they will be outside of the zone and will form three triangles with good geometry.

\subsection{Triangulation}

Triangulation method is one of most popular positioning location techniques in which geometric properties of triangles are used to estimate a node positioning. Basic principle of this method based on three points including two reference node points and a target node point. Basically the triangles will be formed between any two reference nodes and the target node. Triangulation based PL\&T has drawback of the cumulative error during localization and tracking as nodes are continuously tracked at different locations [3]. To get good geometry, we have to carefully place the reference nodes such that there is no angle less than 30 degrees or greater than 150 degrees on the triangles.

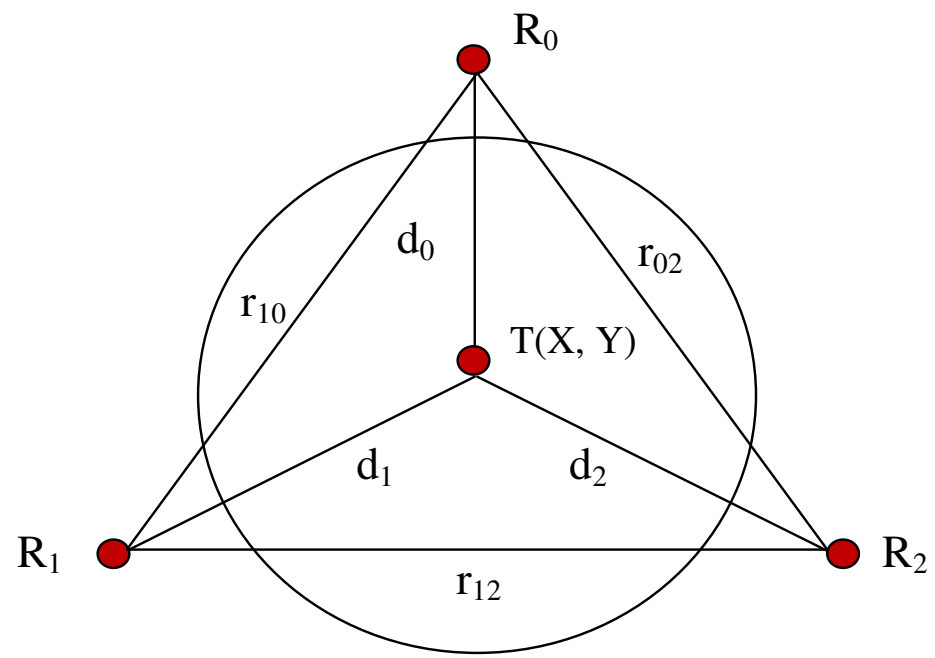

Figure 3. Triangulation with three reference nodes around the predicted zone

After forming the triangles with good geometry we have to determine the range between each reference node and the target node. Figure 3 shows the design of the triangulation with three reference nodes around the predicted zone where $R_{0}, R_{1}, R_{2}$ are three reference nodes and $T$ is the target node that form three triangles; $\Delta R_{0} T R_{2}, \Delta R_{0} T R_{2}$, and $\Delta R_{0} T R_{2}$. The ranges between the target node and the reference nodes will be found by exchanging the IP packets with time stamps of Time of Departure (ToD) and Time of Arrival (ToA). We have to keep record the values of ToA and ToD of each transmission of a packet to find out the transmission time which is the difference between ToA and ToD. For each transmission we can use a set of packets with a known number of packets. We can consider $\mathrm{N}$ number of packets such as $\mathrm{P}_{1}, \mathrm{P}_{2}, \ldots \ldots, \mathrm{P}_{\mathrm{n}}$ will be send from one reference node to target node. For each packet we have to record the time of departure (TOD) such as $t_{1} d_{0}, t_{2} d_{0, \ldots \ldots .,} t_{n} d_{0}$ in the sender side. Again, on the receiving end, we have

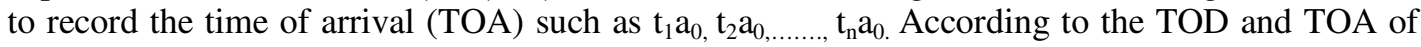
each packet we have to calculate the time difference between TOD and TOA for each packet. Then, we have to calculate the average transmission time by equation 3.5 . 
International Journal of Wireless \& Mobile Networks (IJWMN) Vol. 6, No. 4, August 2014

$$
\text { Average Transmission Time }=\sum_{i=1}^{n} \frac{\left(T O A_{i}-T O D_{i}\right)}{n} \ldots . . . . .
$$

After finding the average transmission time we have to calculate average propagation time by the following equation.

Avg. Propagation Time $=$ Avg. Transmission Time- Avg. Processing Time

We have to use a reference value as a propagation time that relates to an indexed range or distance. By this way, we can get the range $d_{0}$ between the reference node $R_{0}$ and the target node $T$. We need to repeat the above process two times for getting the ranges $d_{1}$ and $d_{2}$ from other two reference nodes $R_{1}$ and $R_{2}$ to the target node $T$ respectively. After getting all the ranges between each reference nodes to the target node, we have to find out the average value of the ranges. Finally, by using the average value of the rages, we will find out the coordinate of the target location $\mathrm{T}(\mathrm{X}, \mathrm{Y})$. As our algorithm will able to track the location of a target node continuously, we have to repeat the overall procedure as well as the computation continuously for predicting the location of the target node in the network. We have to consider each location computed is with respect to a relative origin which is fixed and it has a GPS location. We can even change the relative location to the origin to a GPS value.

\subsection{Dynamic References}

In this research, dynamic references are used for getting more accuracy through geometric triangulation method compare to the basic triangulation method. One of the major concerns of this research is to keep track the moving target as well as stationary target. As the target can move any direction in mobile Adhoc network, it requires dynamic references for applying proper geometry in triangulation method instead of stationary references. When the zone of the target will be predicted with respect a relative origin, reference nodes need to be selected based on their PL\&T information. The nodes that have accurate PL\&T will be perfect as a reference node to form geometry. The accuracy of the triangulation method mainly depends on the proper geometry of the zone prediction and on the reference node selection.

\section{Simulation AND RESUlT}

The $\mathrm{ZF}$ algorithm is based a predictive polynomial of $n$th order where $\mathrm{n}$ has to be at least 3 . It has been implemented is simulation using MATLAB. The general polynomial for the vector distance of the object from a reference point R1 that is shown is Figure 1 is defined by equation 4.1. Reference point is basically a starting point since the polynomial is self-embedded.

$$
X(t)=\sum_{k=0}^{n} a_{k} t^{k} \ldots \ldots \ldots \ldots
$$

Where $n=$ order of the polynomial, $a_{k}$ is the coefficient of the $\mathrm{k}^{\text {th }}$ order of $\mathrm{t}$, and $\mathrm{t}$ is the time at which object is located. $\mathrm{X}(\mathrm{t})$ describes the trajectory of the moving object in time.

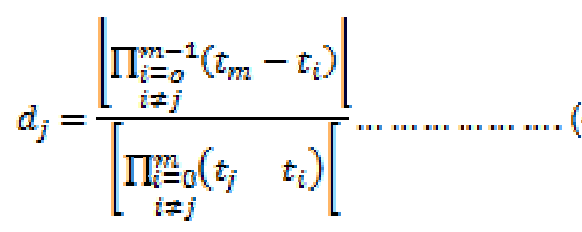


Where $d_{j}$ are the coefficients created for use in computing the vector distance $X(m)$ of the object from the reference point R1 in order to continuously track the object based on the $\mathrm{m}$ immediately preceding locations.

$$
X\left(t_{m}\right)=\sum_{k=0}^{m-1} d_{k} * X\left(t_{k}\right) \ldots \ldots \ldots \ldots
$$

To demonstrate the accuracy of the algorithm, we make the following assumption in equation (4.1): $\mathrm{n}=3 ; \mathrm{a}_{3}=1 ; \mathrm{a}_{2}=-1 ; \mathrm{a}_{1}=2$ and $\mathrm{a}_{0}=5$. Then,

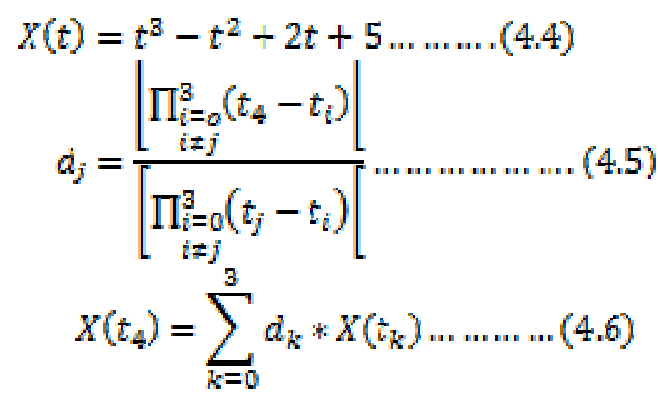

\subsection{Simulation Scenario}

We have simulated two particular scenarios for tracking the position location of a target node. In first scenario, we assume that that target node moves along straight line that means target node does not change the direction of moving. In second scenario, we consider that the target node changes the direction of moving such that target node goes along through the spiral, trapezoidal, or any other way except straight line. For both cases, we have simulated the zone finding (ZF) algorithm and triangulation method to track the position location of a target node for its seven location point. For both cases, in triangulation method four mobile nodes are used in the simulation, where three of them act as reference nodes and one act as a target node. For each instance of time, we have found the zone of the location of the target and then we have placed three reference nodes around the zone by satisfying the condition (Discussed in chapter 3 ) for forming three triangles where each triangle consists of two reference node and the target node. . After forming three triangles, we have sent a particular number of IP packets from each reference node to the target node. By keeping the records value of TOA and TOD of each transmission, we have find out the average transmission time for a particular number of packets from each reference node to the target node by applying equation 3.5. From the average transmission time, we have find out the propagation time by applying equation 3.6.

\subsection{Result for Scenario One}

According to the scenario one, to track the position location of a target, we have run the simulation for zone finding algorithm and for triangulation algorithm. The simulation process and the result we have found are discussed below.

ZF Algorithm: To execute simulation for zone finding, we used MATLAB as a simulation tool and used equations 4.1 to 4.6 for the above example. Equation 4.1 and 4.2 are used for continuously compute the fifth location (for the case $n=3$ ) of the target based on previous four location. As the target moves along the trajectory, when $\mathrm{n}$ goes higher, it will compute the next location by using the higher number of previous location. Table 1 illustrates the accuracy of the algorithm for the case $n=3 ; a_{3}=1 ; a_{2}=-1 ; a_{1}=2$ and $a_{0}=5$ based on our assumption. The first column of Table 1 shows the instance of time t that means on that particular time where the target is 
located. Second column presents the theoretically value of the distances of the target for each instance of time $t$ and third column shows the predicted location distance that we have got from simulation by implementing the iterative process based on having four previous location. We have also find out the error between theoretical value and predicted value of trajectory location of the target that is presented in column four of Table 1.

Table 1. Illustration of the above example data.

\begin{tabular}{|c|c|c|c|}
\hline Time & $\begin{array}{c}\text { Theoretical } \\
\text { trajectory } \\
\text { location }\end{array}$ & $\begin{array}{c}\text { Predicted Location } \\
\text { distance using Iterative } \\
\text { Process }\end{array}$ & $\begin{array}{c}\% \\
\text { Error }\end{array}$ \\
\hline 0 & 5 & - & - \\
\hline 1 & 7 & - & - \\
\hline 2 & 13 & - & - \\
\hline 3 & 29 & - & - \\
\hline 4 & 61 & 61 & 0 \\
\hline 5 & 115 & 115 & 0 \\
\hline 6 & 197 & 197 & 0 \\
\hline 7 & 313 & 313 & 0 \\
\hline 8 & 469 & 469 & 0 \\
\hline 9 & 671 & 671 & 0 \\
\hline 10 & 925 & 925 & 0 \\
\hline
\end{tabular}

The simulation of the algorithm shows high accuracy of prediction of the target location as shown in Table 1. We have plotted the true path (contains the actual locations of the target) and the ZF predicted path of the first 10 locations (over time) of the target using the polynomial $X(t)=t^{3}-t^{2}+2 t+5$ (above). 


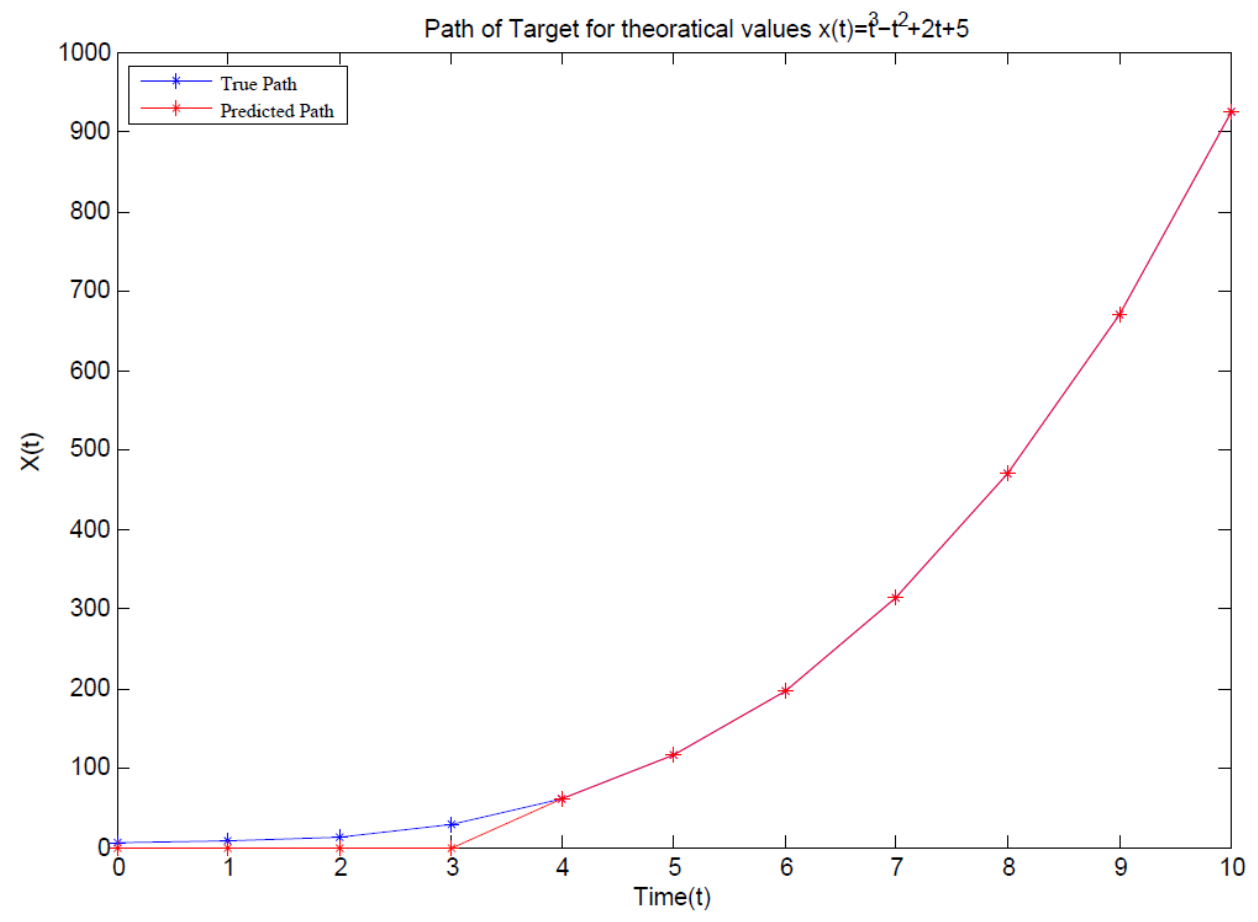

Figure 4. Illustration of accuracy of ZF algorithm

Figure 4 shows the true locations of the target node and compares it with the locations of the predicted value. It shows that the predicted locations at different time instances match closely with those of the true locations. The locations at each time instance is derived from the initial reference point and projects how far the target has moved at each time instance in meters.

Finding the Co-ordinates of the predicted locations: We need to convert the Distance between the initial location point and the current location point of the target node at each instance of time into [X, Y] coordinates. We used equation 3.2 and equation 3.4 (implemented in MATLAB) to convert the distances to the 2-D coordinates. We assume that the first location point's coordinates is $\mathrm{L}_{1}(0,0)$ which makes 30 degree angles along with the $\mathrm{X}$-axis. Table 2 shows the simulation results of findings the $\mathrm{X}-\mathrm{Y}$ coordinates of the first 7 predicted location points below.

Table 2. X-Y coordinates of first 7 predicted location point

\begin{tabular}{|c|c|c|c|}
\hline $\begin{array}{c}\text { Location Point } \\
\text { (Number) }\end{array}$ & $\begin{array}{c}\text { Predicted Location } \\
(\mathbf{m})\end{array}$ & X-Coordinate & Y-Coordinate \\
\hline 1 & 61 & 25.1147 & 14.5000 \\
\hline 2 & 115 & 52.8275 & 30.5000 \\
\hline 3 & 197 & 99.5929 & 57.5000 \\
\hline 4 & 313 & 170.6070 & 98.5000 \\
\hline 5 & 469 & 271.0659 & 156.5000 \\
\hline 6 & 671 & 406.1659 & 234.5000 \\
\hline 7 & 925 & 581.1030 & 335.5000 \\
\hline
\end{tabular}


International Journal of Wireless \& Mobile Networks (IJWMN) Vol. 6, No. 4, August 2014

Triangulation for location prediction within each zone: Once the locations within the zones are identified, the triangulation process is executed to find the exact location. We have used NS-2 as a simulation tool for performing the simulation of triangulation process that consists of several steps. In this simulation, we have found the locations of a target for 7 instance of time for a particular example that we have discussed in section 4.1. We run the simulation for finding 7 location point of a target node. Every time when a target node changes its location, it also dynamically changes all reference nodes' position by satisfying all the condition for making good geometry on triangulation. By applying equation 3.5, we have calculate average Transmission Time, Average Propagation Time for each reference node such as R1, R2, R3 to the target node for $7^{\text {th }}$ location.

We have used a reference value as a propagation time to an indexed range. Here we assume,

\section{1 ns=1 foot or 1 ns $=.3048$ meter}

From this assumption, we have determined the ranges from each reference node to the target location. After getting all three ranges for a target location, we have computed the average value of three ranges for a location point. Table 3 shows the average value of the ranges for 7 location point.

Table 3. Avg. range of the target node from three reference node for 7 location point

\begin{tabular}{|c|c|}
\hline $\begin{array}{c}\text { Target Location } \\
\text { Point }\end{array}$ & Average Range (m) \\
\hline 1 & 9.3113379 \\
\hline 2 & 20.6330321 \\
\hline 3 & 43.5823384 \\
\hline 4 & 82.7503576 \\
\hline 5 & 141.806069 \\
\hline 6 & 225.544281 \\
\hline 7 & 335.408222 \\
\hline
\end{tabular}

Then we have determined the coordinates of the target location by using the average value of ranges. Finally, by taking the average value of the computed coordinates, we have selected the final value of the target coordinates for 7 target location point. Table 4 shows the final value of $X$ $\mathrm{Y}$ coordinates of the target for 7 location point.

Table 4. Value of X-Y coordinates of the target node for 7 location point.

\begin{tabular}{|c|c|c|}
\hline $\begin{array}{c}\text { Target Node } \\
\text { location Point }\end{array}$ & X-Coordinate & Y-Coordinate \\
\hline 1 & 25.1147 & 14.5 \\
\hline 2 & 52.9018333 & 30.5363333 \\
\hline 3 & 99.7072667 & 57.3482 \\
\hline 4 & 170.710933 & 98.1731 \\
\hline 5 & 271.156767 & 156.008033 \\
\hline 6 & 406.166967 & 233.894367 \\
\hline 7 & 581.151233 & 334.7497 \\
\hline
\end{tabular}


According to the value of X-Y coordinate we have plotted a graph in Figure 5 that shows the 7 location points of the target node those are tracked through triangulation method and zone finding method.

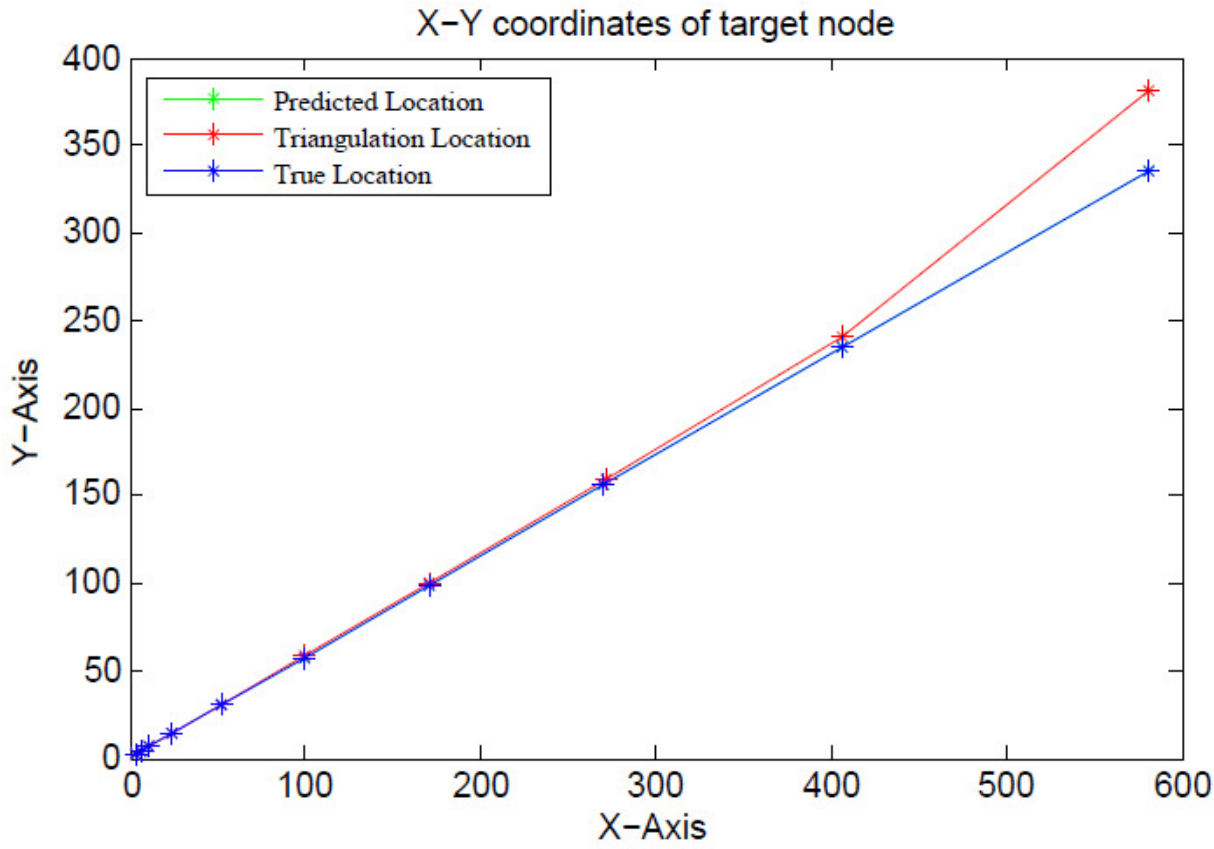

Figure 5. Location Points of the target node those are tracked through triangulation method and zone

\subsection{Results for Scenario Two} finding method.

According to the scenario one, to track the position location of a target, we have run the simulation for zone finding algorithm and for triangulation algorithm. The simulation process and the result we have found are discussed below.

ZF Algorithm: Here, we have considered that the target node moves along a spiral path. Since our ZF algorithm is based on a polynomial equation, it will predict the position location of the target node along with the straight line. Therefore, when the target node changes its direction, through only this $\mathrm{ZF}$ algorithm we cannot predict the position location correctly. For this reason, we need to apply both the ZF algorithm and the triangulation method for tracking the location point accurately. We have followed the same procedure to simulate the $\mathrm{ZF}$ algorithm for this scenario. 


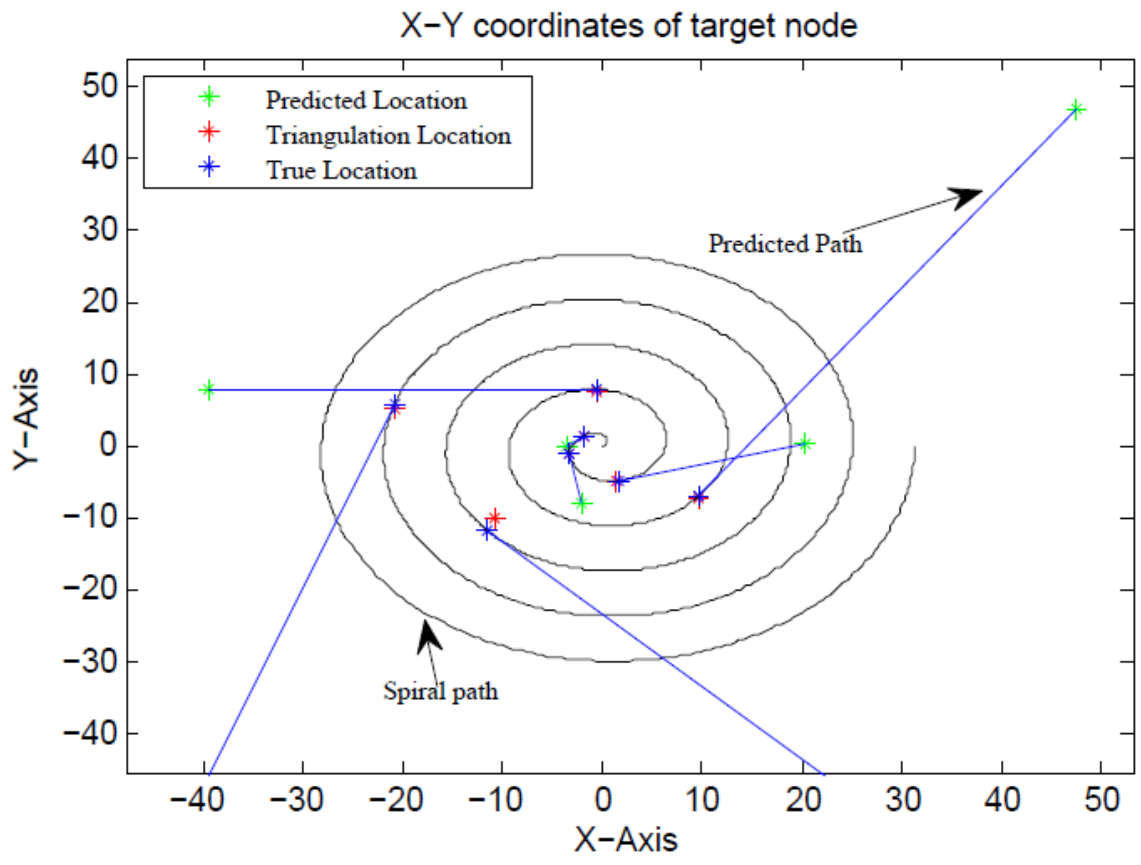

Figure 6. Location points of the target node those are tracked through triangulation method and zone finding method.

Triangulation for location prediction within each zone: Following the same procedure that we have already discussed in 4.4.a. section, we have simulated the triangulation method for find the position location of the target node for its seven location point along the spiral path. Figure 6 shows the location points of the target node those we have found through our implemented ZF algorithm and Triangulation method.

\subsection{Performance Analysis:}

Since we have the true value of X-Y coordinates of a target node's location, we have found out the percentage of error of the triangulation value through equation 4.7. Figure 7 shows the graph of the percentage of the error of the distances according to the true distance value for 7 location point of the target for scenario one. $\mathrm{X}$-axis presents the percentage of the error and $\mathrm{Y}$-axis presents the average distances between the reference nodes and the target node.

$$
\text { Errvr }-\left(\frac{\mid(\text { True Value }- \text { Triangulation Value })]}{\text { True Value }}\right) * 100 \ldots . . . .(4.7)
$$


International Journal of Wireless \& Mobile Networks (IJWMN) Vol. 6, No. 4, August 2014

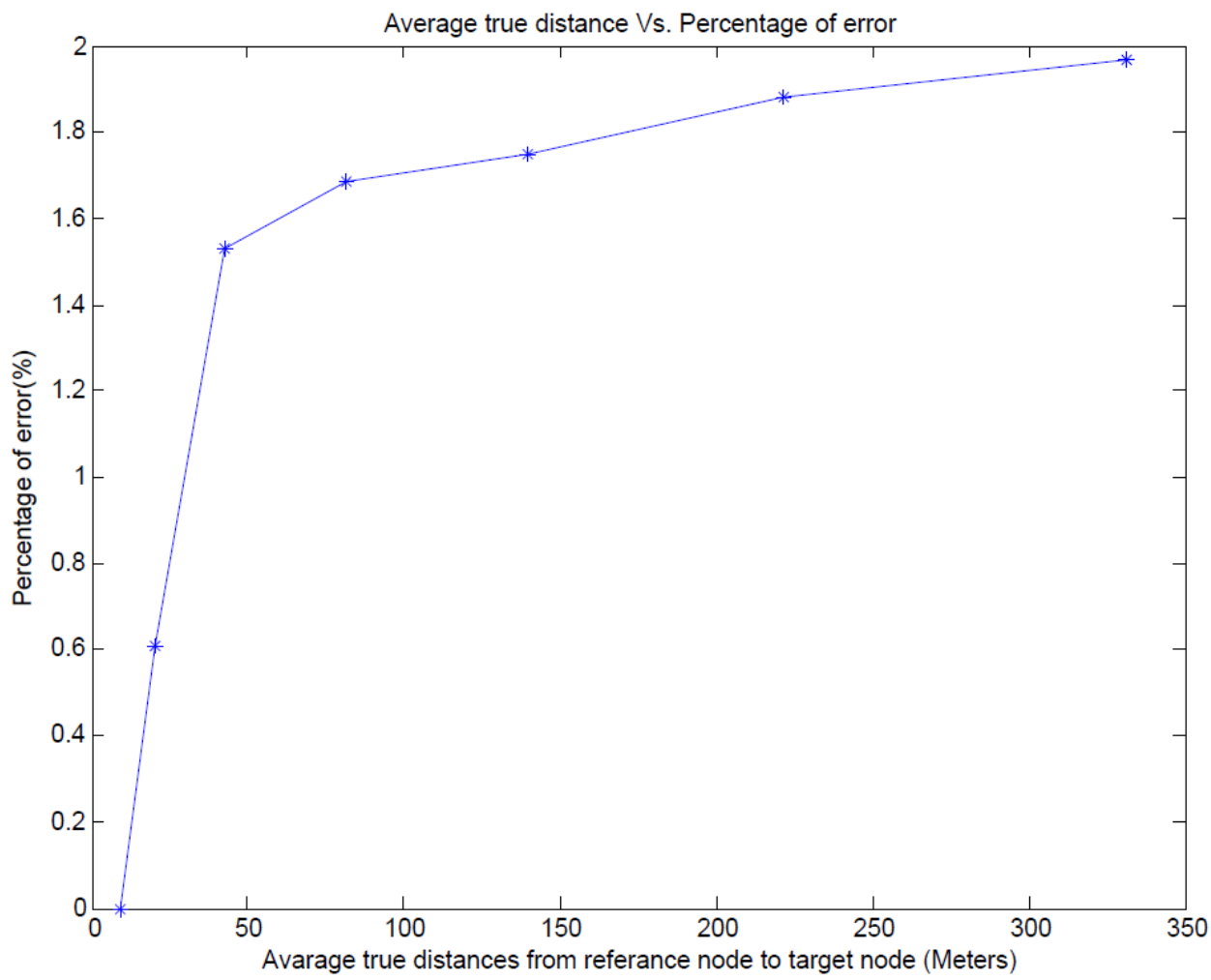

Figure 7. Percentage of the error of the measured distances Vs True distances of the target

We can see that, percentage of error is increasing gradually with average distance value from reference nodes to target node.

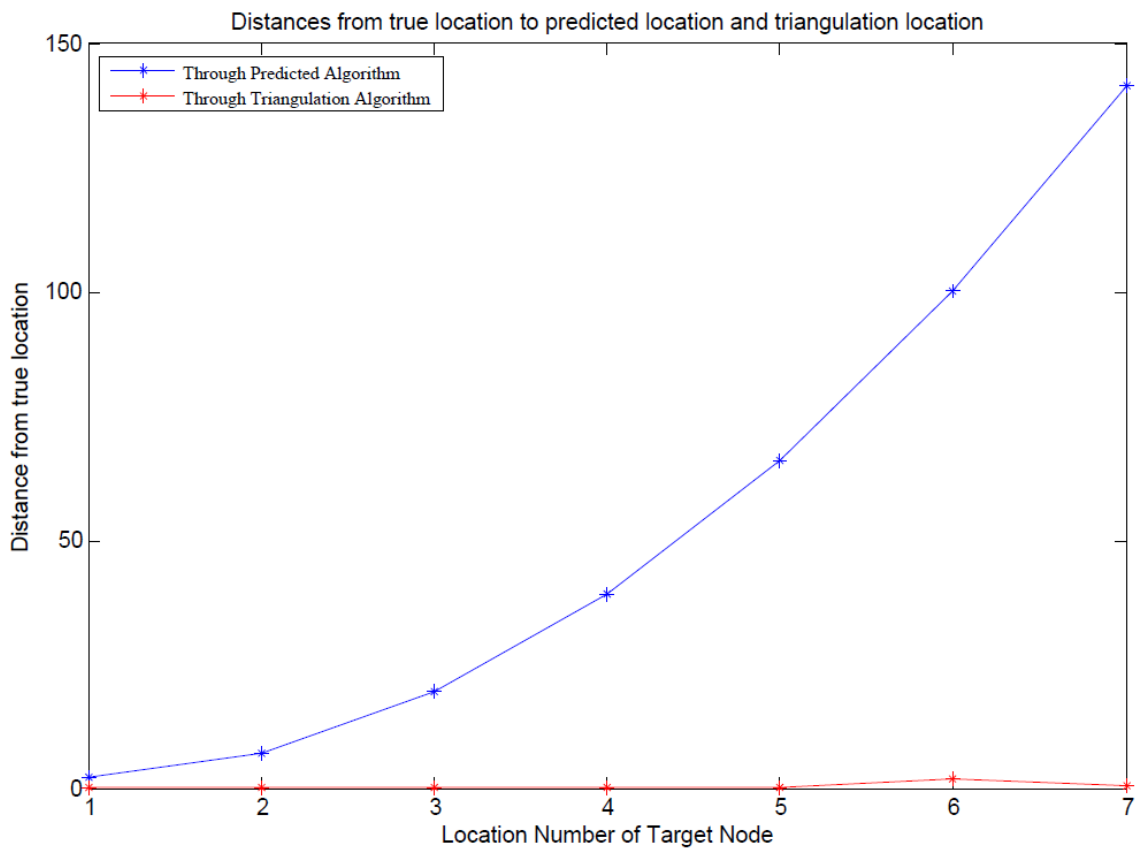

Figure 8 Distances from the true locations of the target node for 7 location points to its predicted location point and triangulation location point. 
International Journal of Wireless \& Mobile Networks (IJWMN) Vol. 6, No. 4, August 2014

Figure 8 shows the graph where we can see the distances from true location point of a target node to its predicted location point and triangulation location point for second scenario. We can see that, in spiral way, the locations those we have found through the ZF algorithm are far away from the true locations of a target node. Therefore, to get the location point accurately, we need to apply triangulation method that help to predict the zone by using reference nodes when target node changes its direction of moving.

\section{CONCLUSION}

In this research, the design of a real time dynamic Position Location \& Tracking (PL\&T) system which is based on integrated predictive zone finding and triangulation method and its performance is shown. The zone finding of a target node is achieved using a predictive algorithm based on knowing previous good PL\&T locations. Once the zone is predicted, the triangulation uses three references with good PL\&T data to be placed around the target node such that the geometry is maintained properly for highly accurate prediction of the PL\&T location of the target node. We use three references for two dimensional PL\&T location. The same thing can be applied for deriving a three dimensional PL\&T location of a target node using four references. This paper limits the discussions only to two dimensional PL\&T location computation. The performance results are provided in this paper which shows highly accurate prediction of the PL\&T location for mobile target nodes.

\section{ACKNOWLEDGEMENT}

This project is partially supported by funding from the National Science Foundation under the research grant NSF 0931679 and support of Research Assistantship from the Department of Electrical and Computer Engineering, Prairie View A\&M University. The authors acknowledge the Mr. Golam Khan in the simulation assistance.

\section{REFERENCES:}

[1] N. Shakhakarmi \& D. R. Vaman, (2012) "Real Time Position Location \& Tracking (PL\&T) using Prediction Filter and Integrated Zone Finding in OFDM Channel", WSEAS transactions in Communications, ISSN:1190-2742, Issue 7, Volume 11.

[2] L. Xiaofeng, W. Fletcher, L. Ian, L. Pietro, \& X. Zhang, (2008) "Location Prediction Algorithm for Directional Communication", Wireless Communications and Mobile Computing Conference, IWCMC '08. International, ISBN: 978-1-4244-2201-2, IEEE Publisher .

[3] N. Shakhakarmi \& D. R. Vaman, (2012) "Dynamic PL\&T using Two Reference Nodes Equipped with Steered Directional Antenna for Significant PL\&T Accuracy”, Wireless Telecommunications Symposium 2012, London, UK.

[4] N. Shakhakarmi \& D. R. Vaman, (2010) "Distributed Position Localization and Tracking (DPLT) of Malicious Nodes in Cluster Based Mobile Ad hoc Networks (MANET)", WSEAS transactions in Communications, ISSN: 1109-2742, Issue 11, Vol. 9.

[5] L. Xiaofeng, W. Fletcher, L. Ian, L. Pietro \& X. Zhang, (2008) "Location Prediction Algorithm for Directional Communication”, Computer Laboratory, University of Cambridge, U.K, and College of Computer Science, Beijing University of Aeronautics and Astronautics, China, IWCMC.

[6] R. Michael Buehrer, Tao Jia \& Benton Thompson, (2010) "Cooperative Indoor Position Location using the Parallel Projection Method", 2010 international conference on indoor positioning and indoor navigation (ipin), zurich, switzerland.

[7] N. Malhotra, M. Krasniewski, C. Yang, S. Bagchi \& W. Chappell, (2005) "Location Estimation in Ad-Hoc Networks with Directional Antennas", School of Electrical \& Compute Engineering, Purdue University, West Lafayette, ICSCS.

[8] R. Siuli, C. Sanjay, B. Somprakash, U. Tetsuro, I. Hisato \& O. Sadao, (2005) "Neighborhood Tracking and Location Estimation of Nodes in Ad hoc Networks Using Directional Antenna: A 
International Journal of Wireless \& Mobile Networks (IJWMN) Vol. 6, No. 4, August 2014

Testbed Implementation", Proceedings of the Wireless Communications Conference, Maui, Hawaii, USA.

[9] B. Zhang \& F. Yu, (2010) "Low-complex energy-efficient localization algorithm for wireless sensor networks using directional antenna," Department of Integrated Electronics, Shenzhen Institutes of Advanced Technology, IET Commun., Vol. 4, pp. 1617-1623.

[10] P. Veeranath, Dr.D.N.Rao, Dr.S.Vathsal \& N.Bhasker, (2013) "Reducing Multipath Effects in Indoor Channel for Analysis of GPS/Pseudolite Signal Acquisition", International Journal of Scientific and Research Publications, Vol. 3, ISSN 2250-3153, url:" www.ijsrp.org".

[11] K. Yedukondalu1, A. D. Sarma \& V. Satya Srinivas, (2011) "Estimation and mitigation of gps multipath interference using adaptive filtering”, Progress In Electromagnetics Research M, Vol. 21, pp.133-148.

[12] R. Ramanathan, (2005) "Antenna Beamforming and Power Control for Ad Hoc Networks", Mobile Adhoc Networking , BBN Technologies, Cambridge, Massachusetts, DOI: 10.1002/0471656895.ch5, url:” http://www.ir.bbn.com/ ramanath/pdf/wiley-bookchap.pdf”.

[13] R. Ramanathan, J. Redi, C. Santivanez, D. Wiggins \& S. Polit, (2005) "Ad Hoc Networking with Directional Antennas: A Complete System Solution”, IEEE J. Sel. Areas Commun., vol. 23, no.3, pp. 496-506.

[14] Gilbert, J.M. \& Won-Joon Choi, (2005) "MIMO Technology for Advanced Wireless Local Area Networks", Design Automation Conference, ISBN:,1-59593-058-2, pp 413 - 415.

[15] A. Pauraj, R. Nabar \& D. Gore, (2003) "Introduction to Space-Time Wireless Communications", Cambridge University Press, Cambridge.

[16] Omnidirectional vs Directional, url "http://www.cisco.com/c/en/us/support/docs/wireless-mobility/ wireless-lan-wlan/82068-omni-vs-direct.html".

\section{Authors}

Sharmistha Khan got the B.Sc. degree in Computer Science from American International UniversityBangladesh (AIUB), Dhaka, Bangladesh in 2006, and the M.S. degree in Electrical Engineering from Tuskegee University, Tuskegee, AL in 2011, respectively. She is currently pursuing her PhD degree in the department of Electrical and Computer Engineering at the Prairie View A \& M University, Prairie View, TX. She is working under the supervision of Prof. Dhadesugoor R. Vaman at Prairie View A \& M University. Her research area includes Mobile Adhoc Network, Cognitive Radio Networks, Sensor Networks, Mobile WiMAX Technology, Handoff Management, and Handoff Performance and Decision Making Algorithms for Broadband Wireless Networks. She is a member of the CEBCOM group at the Prairie View A \& M University.

Dhadesugoor R. Vaman is Texas Instrument Endowed Chair Professor and Founding Director of ARO Center for Battlefield Communications (CeBCom) Research, ECE Department, Prairie View A\&M University (PVAMU). He has more than 38 years of research experience in telecommunications and networking area. Currently, he has been working on the control based mobile ad hoc and sensor networks with emphasis on achieving bandwidth efficiency using KV transform coding; integrated power control, scheduling and routing in cluster based network architecture; QoS assurance for multi-service applications; and efficient network management. Prior to joining PVAMU, Dr. Vaman was the CEO of Megaxess (now restructured as MXC) which developed a business ISP product to offer differentiated QoS assured multiservices with dynamic bandwidth management and successfully deployed in several ISPs. Prior to being a CEO, Dr. Vaman was a Professor of EECS and founding Director of Advanced Telecommunications Institute, Stevens Institute of Technology (1984-1998); Member, Technology Staff in COMSAT (Currently Lockheed Martin) Laboratories (1981-84) and Network Analysis Corporation (CONTEL)(1979-81); Research Associate in Communications Laboratory, The City College of New York (1974-79); and Systems Engineer in Space Applications Center (Indian Space Research Organization) (1971-1974). He was also the Chairman of IEEE 802.9 ISLAN Standards Committee and made numerous technical contributions and produced 4 standards. Dr. Vaman has published over 200 papers in journals and conferences; widely lectured nationally and internationally; has been a key note speaker in many IEEE and other conferences, and industry forums. He has received numerous awards and patents, and many of his innovations have been successfully transferred to industry for developing commercial products. 